\title{
Specific Management of Patients with Acute Abdomen during the COVID-19 Pandemic: A Surgical Perspective from Germany
}

\author{
Florian Kühn $^{\mathrm{a}}$ Matthias Klein ${ }^{\mathrm{b}}$ Henning Laven ${ }^{\mathrm{c}}$ Nikolaus Börner ${ }^{\mathrm{a}}$ \\ Tobias Weinberger $^{\mathrm{e}}$ Florian Streitparth ${ }^{\mathrm{d}}$ Jens Werner ${ }^{\mathrm{a}}$ \\ Tobias S. Schiergens ${ }^{a, b}$ \\ a Department of General, Visceral, and Transplant Surgery, Hospital of the Ludwig Maximilian University of Munich, \\ Munich, Germany; ${ }^{b}$ Emergency Department, Hospital of the Ludwig Maximilian University of Munich, Munich, \\ Germany; ${ }^{\mathrm{C}}$ Department of Anesthesiology, Hospital of the Ludwig Maximilian University of Munich, Munich, \\ Germany; ${ }^{\mathrm{d}}$ Department of Clinical Radiology, Hospital of the Ludwig-Maximilian University of Munich, Munich,

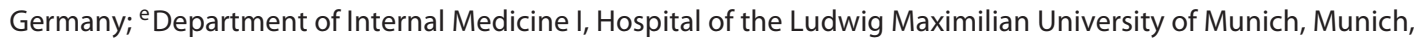 \\ Germany
}

Keywords

COVID-19 · Coronavirus · Triage · Acute abdomen ·

Emergency surgery

\begin{abstract}
During the current COVID-19 pandemic, the triage, assessment, and management of patients presenting to the emergency department with critical conditions has become challenging. The clinical features of COVID-19 are heterogeneous and subtle in many cases. They may easily be overlooked in the case of other acute diseases. Gastrointestinal symptoms are common in patients with COVID-19 as SARS-CoV-2 is able to enter gastrointestinal epithelial cells. However, these complaints can also be caused by a COVID19-independent concomitant abdominal pathology. Therefore, patients with acute abdominal pain and fever need to be assessed very thoroughly. Based on a clinical case, we present our approach of managing emergency patients with acute abdomen and concomitant suspicion of COVID-19.

(c) 2020 S. Karger AG, Basel
\end{abstract}

\section{Clinical Presentation and Triage}

A 59-year-old male patient presented to the emergency department (ED) due to acute abdominal pain. Further history revealed fever, fatigue, headache, and nausea for approximately 5 days. As the fever had been noticed days before the onset of the abdominal pain, the patient was triaged as potentially COVID-19 positive.

When patients present to our ED, a general initial COVID-19 risk assessment at a segregated triage point aims to assign patients to either a locally separated infectious diseases ED (ID-ED) or to the noninfectious ED (NI-ED). Furthermore, all patients are triaged according to the Emergency Severity Index (ESI) [1-3]. Assessment is done by a specially trained triage nurse under the supervision of a physician. These initial items include having had contact with a person with COVID-19 within the last 14 days, a body temperature of more than $37.5^{\circ} \mathrm{C}$ or intake of antipyretics within the last $6 \mathrm{~h}$, respiratory symptoms such as cough or dyspnea, and a new absence of olfaction. If 1 item (or more) is positive or a patient cannot be assessed for all items (e.g., due to dementia or loss of consciousness), he/she is first treated at the ID-ED. All patients who are found to bear a risk for COVID-19 are further treated in separated single rooms with contact precautions (patient: surgical face mask; staff: FFP2 mask, 
Table 1. Comparison of typical findings in COVID-19 patients with those in the present case

\begin{tabular}{|c|c|c|}
\hline Parameters & Typical COVID-19 & Present case \\
\hline \multicolumn{3}{|l|}{ Patient characteristics } \\
\hline Age, years & $\sim 70$ & 59 \\
\hline Sex & Male & Male \\
\hline Most common comorbidity & Obesity and hypertension & None \\
\hline \multicolumn{3}{|l|}{ Symptoms } \\
\hline Fever & Common & Yes \\
\hline Cough & Common & No \\
\hline Shortness of breath & Common & No \\
\hline Tiredness, aches & Common & No \\
\hline Sore throat & Sometimes & No \\
\hline Loss of smell or taste & Sometimes & No \\
\hline Diarrhea & Common & No \\
\hline Nausea & Common & Yes \\
\hline Vomiting & Sometimes & Yes \\
\hline Abdominal pain & Seldom & Yes \\
\hline Gastrointestinal bleeding & Seldom & No \\
\hline \multicolumn{3}{|l|}{ Laboratory parameters } \\
\hline Lymphopenia & Common & Yes \\
\hline Eosinopenia & Common & Yes \\
\hline Procalcitonin & Normal $(<0.15 \mathrm{ng} / \mathrm{mL})$ & Increased \\
\hline CRP & Slightly elevated & Markedly elevated \\
\hline Interleukin-6 & Slightly elevated & Markedly elevated \\
\hline Hypoalbuminemia & Common & Yes \\
\hline Elevated ferriti & Common & Yes \\
\hline \multicolumn{3}{|l|}{ Radiologic chest findings } \\
\hline \multicolumn{3}{|l|}{ Conventional $\mathrm{x}$-ray } \\
\hline Pulmonary parenchymal opacities & Common & Mild \\
\hline \multicolumn{3}{|l|}{ HR-CT } \\
\hline Ground-glass opacity & Common & Mild \\
\hline Consolidation & Common & Mild \\
\hline Bilateral distribution & Common & Yes \\
\hline Posterior lungs & Common & Yes \\
\hline Peripheral lungs & Common & Yes \\
\hline Air bronchogram & Common & No \\
\hline Reticulation, bronchiectasis & Sometimes & Yes \\
\hline Pleural effusion & Seldom & No \\
\hline Pericardial effusion & Seldom & No \\
\hline Lymphadenopathy & Seldom & No \\
\hline
\end{tabular}

glasses, gloves, and gown) until COVID-19 can be ruled out. Accordingly, the presented patient received a triage index score ESI 2 and entered the ID-ED area.

\section{Diagnostic Strategy}

Nasal and pharyngeal swabs were taken for further assessment including PCR-based testing for SARS-CoV2-RNA N-gene-1. The laboratory findings are shown in Table 1 and they are compared to typical findings in COVID-19 patients. They show markedly elevated levels of CRP $(17.3 \mathrm{mg} / \mathrm{dL})$ and interleukin $6(2,166 \mathrm{pg} / \mathrm{mL})$, lymphocytopenia $(0.20 \mathrm{G} / \mathrm{L})$, eosinopenia $(<0.01 \mathrm{G} / \mathrm{L})$, and hypoalbuminemia, and an increase in serum ferritin $(2,125 \mathrm{ng} / \mathrm{mL})$.

High-resolution computed tomography (HR-CT) of the thorax and abdomen was conducted immediately (Fig $1 \mathrm{~A}, \mathrm{~B})$ as PCR results were pending but fever and eosinopenia made COVID-19 likely. Our ID-ED unit comprises separate X-ray equipment and CT scanners to ensure a quick workup despite the necessity of contact precautions. CT revealed an intra-abdominal abscess and moderate amounts of free intra-abdominal air, probably due to a perforated jejunal diverticulum. Thoracic CT resulted in mild ground-glass opacity as well as bronchiectasislike changes in both inferior lobes, which was considered suspicious for COVID-19 (Fig. 1B) [4]. 
Fig. 1. Findings of a patient with COVID19 presenting with an acute abdomen (AC). A Abdominal contrast-enhanced CT on the coronal plane showing an intestinal perforation with free air (red arrow) as an intra-abdominal septic focus. B Axial HR$\mathrm{CT}$ of the lung showing bronchiectasis (green arrows) of both inferior lobes but also mild bilateral parenchymal involvement with peripheral patchy ground glass (yellow arrows; ${ }^{*}$ diaphragm) and few posterior consolidative (blue arrow) pulmonary opacities suspicious for an early stage of COVID-19. C Intraoperative finding of a perforated jejunal diverticulum. D Postoperative chest X-ray showing unspecific basal compactions (arrows).

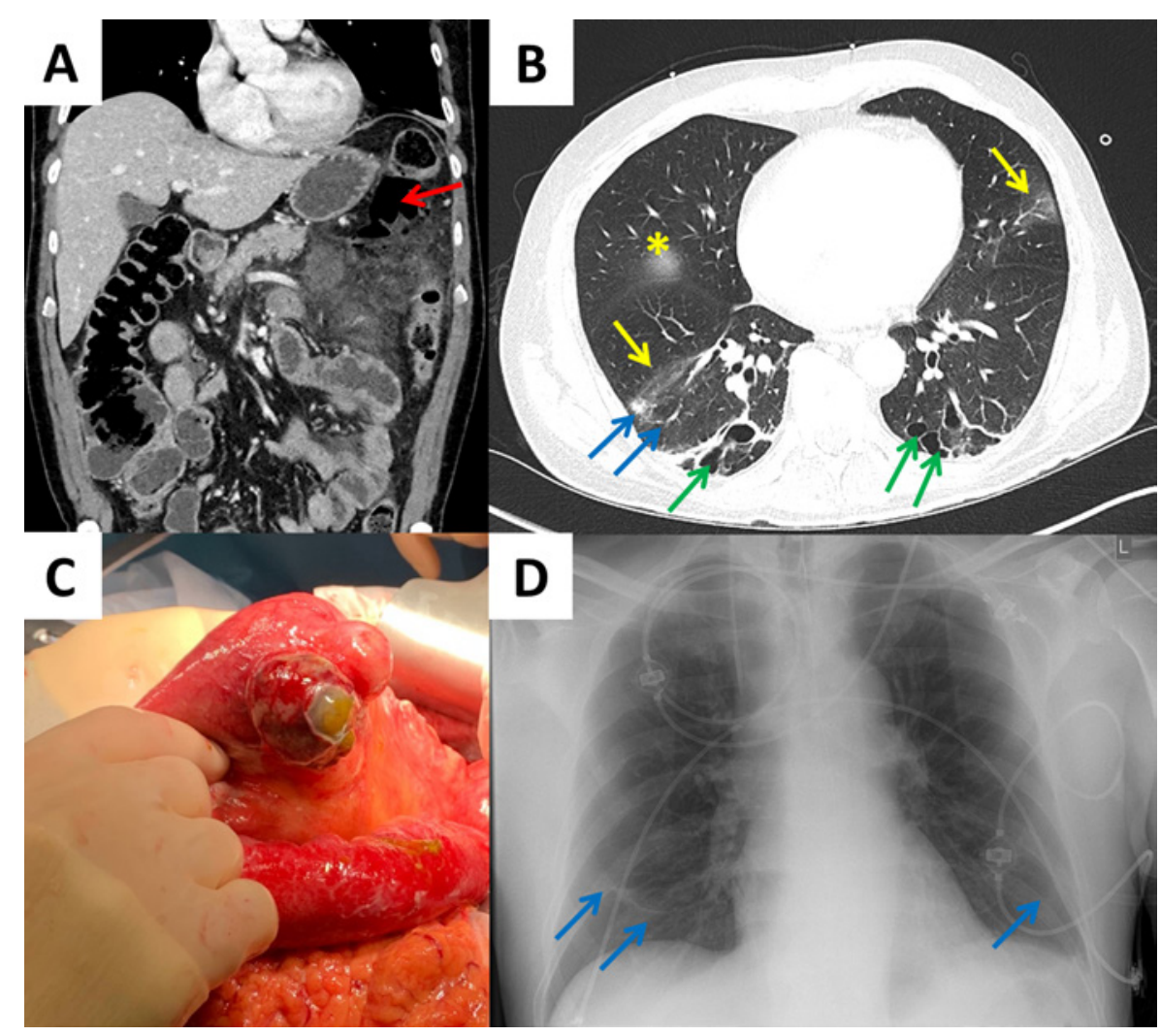

\section{Management in the OR and Safety Measures}

Based on those findings, emergency surgery for the intestinal perforation was indicated. Since COVID-19 could not be ruled out and the result for the swabs were not expected before the next day, all COVID-19 safety measures were continued during further management.

Patients who are suspicious for COVID-19 or have confirmed diagnosis are transported under strict isolation measures in COVID-19-designated escalators and corridors where possible. During induction of anesthesia, safety measures include wearing FFP3 masks, the use of virus-tight HME filters in the ventilation systems, and the rapid sequence induction procedure for intubation with primary use of video laryngoscopy. Operations are conducted in a specifically designated COVID-19 operating room with ventilation-technical equipment facilitating a negative air pressure. The intraoperative safety measures are based on currently available recommendations (i.e., from the American College of Surgeons) and include FFP2 masks, safety glasses/face shields, double surgical gloves, a water-proof gown, shoe covers, performing an open surgical procedure, maximum reduction of aerosols (e.g., avoiding electrocautery and high-speed tools, double aspiration) [5-7]. In the present case, we found a perforated jejunal diverticulum with an abscess and local peritonitis (Fig. 1C); an open small-bowel segmental resection and anastomosis were performed. Following the operation, the patient remained intubated and was conveyed to one of our designated COVID-19 intensive care units. The day after the operation, the SARS-CoV-2-RNA test from nasal and pharyngeal swabs turned out to be positive. A bedside portable chest radiograph on postoperative day 1 showed slight basilar opacities in both lungs (Fig. 1D), consistent with the findings on chest CT on admission.

On postoperative day 3 , the patient was transferred to one of our COVID-19 intermediate care units. Here, SARSCoV-2-RNA was detected in a postoperative PCR-based stool analysis, whereas it was not present in swabs taken from intra-abdominal drains. Sputum analysis on postoperative day 8 turned out to be still positive. The completely asymptomatic patient was discharged 10 days after the initial surgery and put in home quarantine for 14 days.

\section{Discussion and Conclusion}

The present case and our standardized assessment, triage, and management process emphasize the importance of a "safety first" principle in the management of patients presenting with an acute abdomen during the COVID-19 pandemic in order to reduce risks for patients and medical staff. Prehospital triage and in-hospital safety measures must begin at the first patient contact. Our presented case and its management show that the assessment of patients 
with an acute abdomen is challenging due to overlapping of typical and atypical symptoms as well as laboratory and radiologic findings, depending on the disease stage. Furthermore, high SARS-CoV-2 RNA concentrations and sgRNA-containing cells could be detected in the stool of infected patients $[2,8]$, indicating active replication in the gastrointestinal tract in analogy with SARS-CoV, which was shown to be excreted in stool, from which it could be isolated. This may be of significance especially in gastrointestinal perforations regarding potential transmission and infection control intra- and postoperatively [2].

If there is any suspicion of COVID-19, all safety measures should be rigorously applied and continued until proof of the contrary is obtained. An obvious intra-abdominal septic focus with a concomitant fever does not rule out a COVID-19 infection. Currently, the same holds true for any other medical condition when any signs of COVID-19 are present. Besides, also asymptomatic carriers are a problem for the health care system. At the time point when testing becomes more readily and widely available, testing of all patients admitted to the hospital will need to be considered.

\section{Statement of Ethics}

No studies on humans or animals were performed. The patient data of this report was collected retrospectively and treated anonymously.

\section{Conflict of Interest Statement}

The authors declare that no conflict of interests exists.

\section{Funding Sources}

No funding was received for the present report.

\section{Author Contributions}

F.K. and T.S.S. designed this report, acquired data, interpreted data, and wrote this paper. M.K., H.L., N.B., T.W., F.S., and J.W. interpreted and discussed data and critically revised this paper for intellectual content. All of the authors revised and approved this work for publication.

\section{References}

1 Tian Y, Rong L, Nian W, He Y. Review article: gastrointestinal features in COVID-19 and the possibility of faecal transmission. Aliment Pharmacol Ther. 2020 May;51(9):843-51.

2 Wong SH, Lui RN, Sung JJ. Covid-19 and the digestive system. J Gastroenterol Hepatol. 2020 May;35(5):744-8.

3 Kuriyama A, Urushidani S, Nakayama T. Five-level emergency triage systems: variation in assessment of validity. Emerg Med J. 2017 Nov;34(11):703-10.
4 Chung M, Bernheim A, Mei X, Zhang N, Huang $\mathrm{M}$, Zeng $\mathrm{X}$, et al. CT Imaging Features of 2019 Novel Coronavirus (2019-nCoV). Radiology. 2020 Apr;295(1):202-7.

5 Brindle ME, Gawande A. Managing COVID-19 in Surgical Systems. Ann Surg. 2020 May; DOI: 10.1097/SLA.0000000000003923.

6 Ross SW, Lauer CW, Miles WS, Green JM, Christmas AB, May AK, et al. Maximizing the Calm before the Storm: Tiered Surgical Response Plan for Novel Coronavirus (COVID-19). J Am Coll Surg. 2020 Jun;230(6): 1080-1091.e3.
7 Forrester JD, Nassar AK, Maggio P, Hawn MT. Precautions for operating room team members during the COVID-19 pandemic. J Am Coll Surg. 2020 Apr;230(6):1098-101

8 Wölfel R, Corman VM, Guggemos W, Seilmaier M, Zange S, Müller MA, et al. Virological assessment of hospitalized patients with COVID-2019. Nature. 2020 May;581(7809): 465-9. 